\title{
Agent Technology for Multi-criteria Regulation in Public Transportation
}

\author{
Nabil Morri, Sameh Hadouaj, and Lamjed Ben Said
}

\begin{abstract}
This paper provides an agent technology for a decision support system. This system is designed to detect and regulate the traffic of multimodal public transport when many disturbances come simultaneously. The objective of this system is to optimize the regulation action by learning technique of regulator. The goal of this research is to improve the quality of public transport service provided to users and respect the use rules (safety rules, business rules, commercial rules, etc.). So, to improve the quality service of the user, we have to optimize simultaneously several criteria like punctuality, regularity and correspondence in disturbance case. In this paper, we focus primarily on a multi agent system for optimizing and learning of Regulation Support System of a Multimodal Public Transport (RSSPT). We have validated our strategy by simulating situation related to existing transportation system.
\end{abstract}

Index Terms-Multi agent system, learning agent, optimization, regulation support system, intelligent transportation system.

\section{INTRODUCTION}

Today, millions of users daily use transportation systems. In urban areas with high population density, these systems convey a very large population and become an indispensable service in people's lives. Also, the increasing use of the vehicle, pose a serious problems in the social, economic and environmental terms. The public transport has become a major issue of collective and daily life. Thus, with the amplification of public transport systems, traffic management becomes more complex. This complexity is due to the presence of several transportation modes (multi-modality) as metro, bus and tram, as well as the existence of elements with different natures like vehicle, station, routes, etc.

With the development of information technology, transport operators are seeking effective solutions to improve

Manuscript received December 20, 2015; revised February 15, 2016. This work was supported by the Emirates College of Technology and Tunis University (Université de Tunis).

Nabil Morri is with the Emirates College of Technology and Tunis University, Tunisia (e-mail: morrynabil@gmail.com).

Sameh Hadouaj is with Higher Colleges of Technology of Abu Dhabi and Tunis University, Tunisia (e-mail: hadouaj@yahoo.fr).

Lamjed Ben Said is with Tunis University, Tunisia (e-mail: lamjed.bensaid@isg.rnu.tn). the service quality provided to public transport users. In fact, the system should consider easily these solutions and maintain as more as possible the public transportation traffic by respecting the theoretical timetable of vehicles. We can encounter perturbations that deteriorate the quality service of the public transport. These perturbations are due to the emergence of complex and random phenomena like accident, congestion of the traffic, etc. In order to regulate the traffic of public transport, the system should optimize the three criteria punctuality, regularity and correspondence. Therefore, it is necessary to provide a regulation support system that can detect on real time a disturbance and regulate the traffic of public transport. Hence, our system takes into account the major criteria that have to be optimized in a traffic regulation: punctuality, regularity, and correspondence [1]. It also deals simultaneous many disturbances. The regulation public transport domain is highlight dynamic, open and complex. Therefore, multi-agent system is the suitable approach used to model such system requirements.

This paper is organized as follows. Section II presents the existing works with their limits. Section III describes the regulation of public transport. Section IV gives an overview of the optimization problem for regulation process. Section V explains Multi-agent modeling and details the agent technology in its three levels such as organization, intra-agent, and interaction level. Section VI proves the efficiency of our strategy by an experimentation and result. Finally, Section VII gives conclusion and some perspectives.

\section{EXISTING WORKS}

The existing regulation support systems of public transport have limits. Firstly, the majority of existing systems don't ensure the two fundamental tasks of regulation such as detection and decision [1]-[3]. They focus essentially only on the decision phase without taking into consideration the detection phase. They use only alarms as complementary information received from operator.

Secondly, They don't take into account perfectly the major criteria that have to be optimized in a public transport regulation: punctuality, regularity and correspondence similar to approaches that address the regulation of traffic lights [4]-[6]. Also they don't address the public multimodal transport (bus, metro and tram) and can't handle multiple disturbances simultaneously like some mathematical approach [7], [8]. While the evolutionists approaches [9], [10] gives only synthetic result and don't actually give regulation based on all criteria. Also, the majority of works don't ensure a follow up of the regulation action impact in order to update the information system on real-time and develop its expertise in the regulation process. Hence, our 
goal is to build Regulation a Support System for Public Transport (RSSPT) that overcomes these limits.

The multi-agent paradigm is well suitable to design transport applications. Indeed, when implementing these applications in highly dynamic and constrained environments, it is very important to use method that offers communication, collaboration and negotiation between heterogeneous stake holders.

\section{Regulation Process FOR Decision MAKING}

We characterize the regulation process of public transport traffic as a system that diagrammed as Fig. 1.

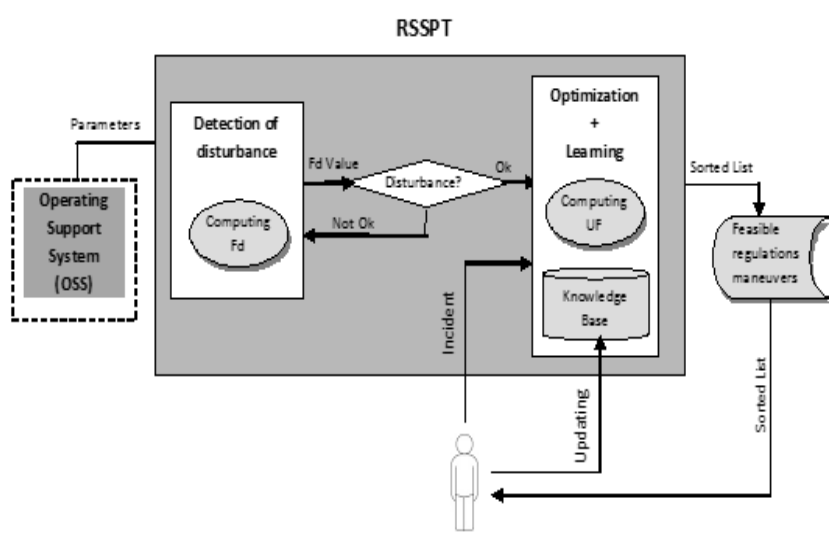

Fig. 1. Regulation process in RSSPT.

The operation Support System (OSS) contains the information that gives a detailed vision of public transport traffic in real time. It communicates them as parameters to the Regulation Support System of Public Transport (RSSPT). These parameters cover the vehicle properties (position, mode, type, line, driver...), frequency, peak hours (factory exit, school exit, ceremony...), holiday, etc. To detect disturbances, the RSSPT calculates continuously the detection function value $F_{d}$ with simultaneous consideration of punctuality, regularity and correspondence criteria. If $F_{d}$ value falls down into a critical zone, the optimization and learning phase will be started. The critical zone is defined by experts of public transport traffic according to the treaty region of the network traffic.

To ensure optimization and learning tasks, our system calculates the value of the objective function named utility function $U F$. This function use some parameters received from OSS and complementary information provided by the operator like position and the cause of disturbance (vehicle breakdown, exceed parking time, uneasiness driver, traffic congestion, etc.). Also, it takes into account some constraints like not exceed the allowed working time for vehicle and time load for driver per day.

After that, the RSSPT produces traffic diagnostic of the network current state. According to this diagnostic, it establishes solutions and takes the adequate decision like decreasing the number of vehicle provided, accelerating or decelerating vehicle, deriving vehicle, etc. In this phase, the operator understands the progress of the current disturbance and its potential impact on network traffic. Finally, the operator gives the suitable decision and controls theirs consequences on public transport network to update the system knowledge base for learning task.

\section{MATHEMATiCAL MOdEL FOR OPTIMIZATION PROBLEM}

Optimization problems are real world problems we encounter in many areas. In the intelligent transportation, we formulate mathematical description called mathematical model to represent the regulation situation problem [11]. The model consists of following components:

\section{A. Decision Variables}

1) Punctuality criteria: The criteria value $V p$ as follows:

$$
V_{p}=V_{R_{-} v}+\Delta_{R t}
$$

With $\Delta_{R t}$ is the remaining time needed to coming for vehicle passage. Also, $V_{R_{-} v}$ signifies the waiting time of each coming vehicle. It is equal to:

$$
V_{R_{-} v}=T_{C}-T_{T_{-} v}
$$

With $T_{C}$ is the Current time and $T_{T_{-} v}$ is the theoretical time of coming vehicle. We note that remaining time is calculated by this formula:

$$
\Delta_{R t}=\sum_{i=1}^{n}\left(S_{i, j_{-} v}+\text { Stop }_{i_{-} v}\right)
$$

With $S_{i, j_{-} v}$ is the needed time to travel from station $i$ to station $j$ for vehicle $v$. This needed time must take into account the traffic state and the transportation mode. It should be based on all parameters values received from OSS. Here Stop $_{i_{-} v}$ is the stop time in station $i$ for vehicle $v$. The total number of stations before coming to station $S$ is $n$.

2) Regularity criteria: The criteria value $V r$ as follows:

$$
V_{r}=V_{p}-T_{R_{-} v_{-} \text {behind }}
$$

The $T_{R_{-}{ }_{-}}$behind is the real time of behind vehicle passage (see Fig. 2).

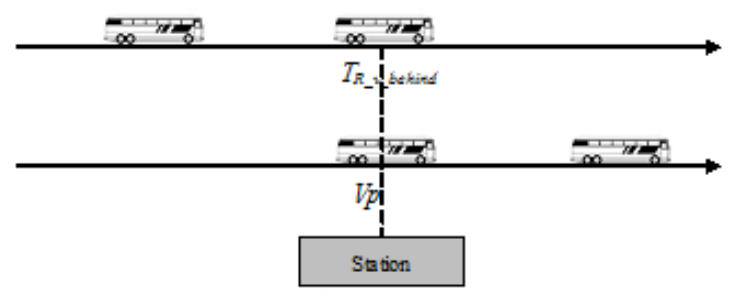

Fig. 2. Calculation of the regularity criteria value.

When $V_{r}$ value is less than vehicle frequency, it means that there is a risk of a vehicle train. Otherwise we deduce that there is a risk of a gap. These risks are estimated by experts of the system.

3) Correspondence criteria: the criteria $V c$ is equal to:

$$
V_{c}=\sum_{i=1}^{n} f_{i}\left(\Delta_{i_{-} \nu_{-} c}\right)
$$

Here $f_{i}$ represents the waiting time impact factor of $i$ th corresponding vehicle. This factor indicates the importance weight of the corresponding vehicles in the calculation of the regulation solution. It is necessary that $\sum \quad f_{i}=1$ with $n$ is the number of the corresponding vehicles. And $\Delta_{i_{-} v_{-} c}$ represents the waiting time of $i$ th corresponding vehicle. It is equal to:

$$
\Delta_{i_{-} \nu_{-} c}=V_{p}+\Delta_{R t_{-} i}
$$

Here $\Delta_{R t_{-} i}$ represents the remaining time of the $i$ th corresponding vehicle passage in the transfer station.

\section{B. Objective and Decision Functions}

Objective function: The objective of this problem is 
expressed by optimizing the utility function $U F$ for each vehicle. We calculate the value of utility function according to three criteria: punctuality, regularity and correspondence.

$$
U F=W_{p} . V_{p}+W_{r} \cdot V_{r}+W_{c} \cdot V_{c}
$$

Here the weights $W_{p}, W_{r}$ and $W_{c}$ are relative to the importance of the different criteria. E.g. punctuality for school bus is more important than the passenger bus, against keeping good correspondence is more interesting for passenger bus than school bus. The weights values are between 0 and 1 and $\sum W_{i}=1$ with $i \epsilon\{p, r, v\}$.

Detection function: This function takes into account the three criteria punctuality, regularity and correspondence without considering the remaining time of the coming vehicle. It based only on the waiting time $\left(V_{p}=V_{R_{-} v}\right)$. Consequently, it is equal to:

$F_{d}=\left(W_{p}+W_{r}+\sum_{i=1}^{n} W_{c}\right) . V_{R_{-} v}-\left(W_{r} \cdot T_{R_{-} v_{-} b e h i n d}+W_{c} \cdot \sum_{i=1}^{n} T_{i_{-} v_{-} c}\right)$

\section{Constraints}

Working time for driver: The regulation system should take into account the working time reminding for the driver. When our system chooses the regulation action, the time needed to achieve this action should not exceed the reminding working time for driver.

$$
T_{f d}-T_{C} \leq T_{r d}
$$

With $T_{f d}$ is the time when the driver achieves its trip after regulation and $T_{r d}$ is the working time reminding for driver.

Working time for vehicle: To keep the good state of vehicles, the regulation system should not exceed the allowed vehicle load on a day.

$$
T_{f v}-T_{C \&} \leq T_{r v}
$$

With $T_{f v}$ is the time when the vehicle achieves its trip after regulation and $T_{r v}$ is the working time reminding for vehicle.

\section{Multi-Agent Modeling}

\section{A. Multi-agent System for Regulation Support System}

Multi-agent modeling can give a suitable solution to multimodal public transport network activities where autonomous entities, called agents, interact with each other in a distributed, open, dynamic, heterogeneous and complex environment [11]. We note that multi-agent systems are increasingly present in the field of traffic regulation [12], [14] The advantage of this approach is the explicit representation of processes that it models. The regulation public transport is distributed because information are geographical dispersed over the network. Dynamic, where there is daily change of information, for example, vehicle can move forward, slow down, accelerate and communicate its passage with other agents like OSS and stations. Open, where the state of public transport networks changes continuously. It must manage agents who can enter and exit freely, for example add a large number of vehicle agents to simulate congestion situation. Also it is heterogeneous where the actors of our system are varied: vehicle, station, regulator, etc. Moreover, it is complex when it requires entities with complex nature. These entities can reason and communicate via messages to solve problem. As a consequence, our RSSPT modeling is based on agents have a different behaviors and able to communicate and cooperate to detect and resolve disturbances.

\section{B. Agent Technology}

Organization Level: We describe the multi-agent architecture of our regulation system in Fig. 3.

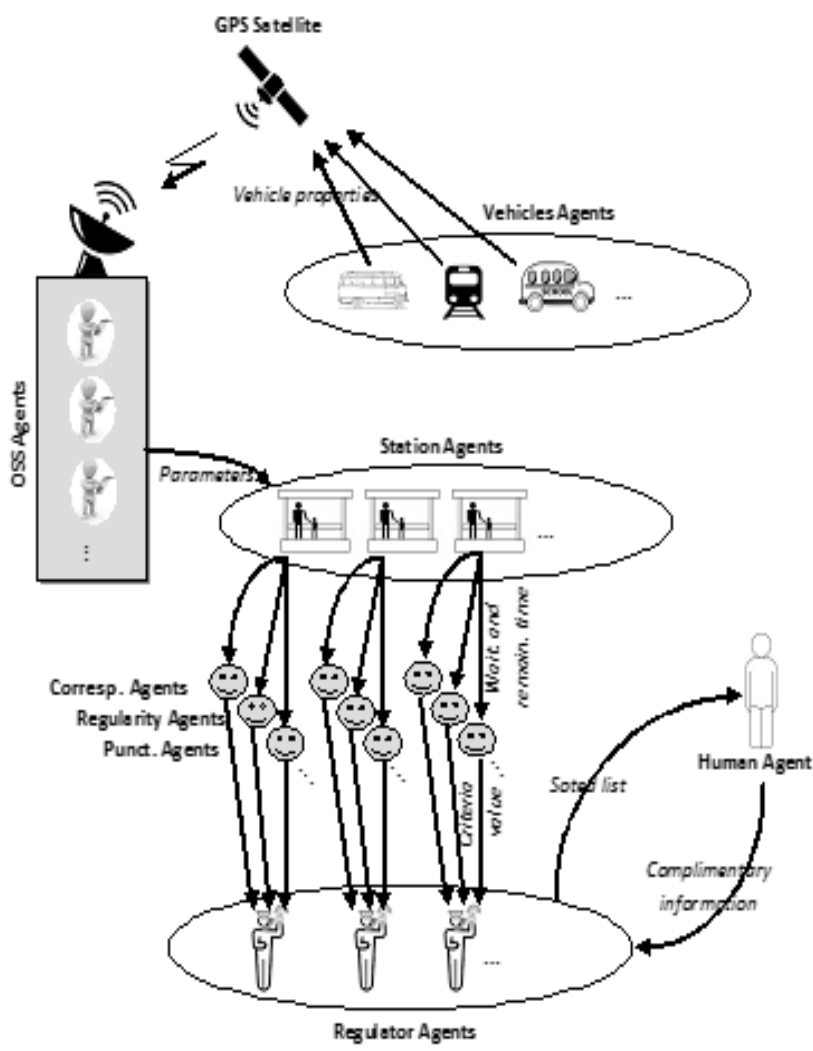

Fig. 3. Multi-agent architecture of RSSPT.

Our system contains varieties agent class: vehicle, OSS, station, criteria (punctuality, regularity, correspondence...), regulator and human. They function in permanence. At first, it processes the disturbance detection every lap's time. It use the information stored in OSS agents. This information represents all properties of vehicle (type, mode, driver, position, charge, working time, line...) and the traffic state of the treated region. The station agent receives the necessary information from OSS agent, calculates and sends to each criteria agent the waiting time to indicate that the treated vehicle is not yet coming. The criteria agent of the concerned vehicle calculates its value. The regulator agent uses these values to detect disturbance after computing the detection function value $F_{d}$. When there is a disturbance, the station resends remaining time to optimize the utility function $U F$. Finally, the human agent chooses adequate regulation maneuver from sorted regulation actions list offered by the regulator agent.

A unique characteristic of this model is that the same agents are used to ensure the detection and the regulation process (optimization and learning) of our RSSPT. This makes the model simpler and solves the problem of data duplication. We can also update the list of criteria without modifying the model. We have to just add other criteria agent able to calculate its criteria value and send it to the regulator agent. In this case the computing of the utility function value should take into account only the new criteria list values with theirs weighs. 
In addition this architecture detects the disturbance on time and ensures self-organization among the different agents to achieve a collective goal. Furthermore, the regulation can deal with many disturbances at the same time and use its expertise by two types of information: complimentary information as incident (source and position) received from human agent and real traffic condition as parameters received from OOS agent.

1) Intra-agent Level: So far we have presented multi-agent architecture by describing its functionality. It models agents having common goal that is regulation of public transport. Now, we will have to explain how the agents work by designing the agent program. In our system we describe agents into four main groups: reflex agent, Model-based Reflex agent, utility-based agent and learning agent. They will have almost the same skeleton, namely, accepting percepts from an environment and generating actions following reasoning task (see Fig. 4). The functioning of reasoning task depends on the agent group.

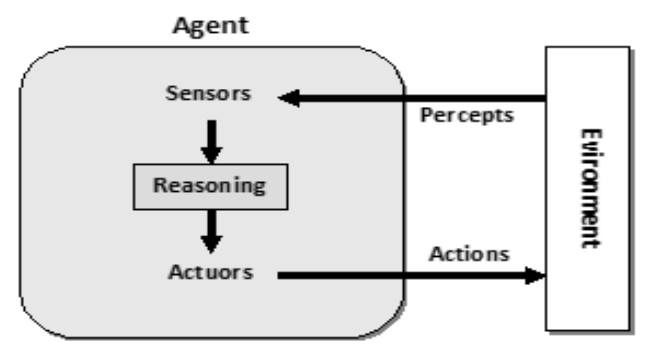

Fig. 4. Intelligent agent skeleton.

a) Simple Reflex agent: It uses condition-action rules. This part shows how the condition-action rules allow the agent to make the connection from percept to action.

- Vehicle agent: At the start service, each vehicle creates a corresponding OSS agent and deletes it at the end of its service. It is characterized by mode (bus, metro or tram), position, speed, capacity, number of passengers, line which affected, mission (academic, commercial or passenger), driver, remaining work time, etc. It gives to the corresponding OSS agent via GPS its properties with position and number of passengers to descend at the next station for computing of the stop time.

- Criteria agent (Correspondence, Regularity, and Punctuality): If the agent receives the waiting or remaining time of corresponding vehicle from station agent, it calculates its criteria value. After that, it sends the result to the Regulator agent for detecting disturbance and optimizing the regulation action.

b) Model-based Reflex agent: Some actions require agents have to keep track of an internal state. That depends on the percept history and needs two type of knowledge such as how the environment evolves independently of the agent? And how the agent's actions affect the environment?

- OSS agent: It receives necessaries properties from vehicle and selects all station agents related to line of the vehicle. After that it collects the complimentary information of the traffic state from its internal state.
Later it formulates parameters and sends it to all associating station.

- Station agent: It is related to one or more lines. Each agent must construct and memorize in its internal state all theoretical times and real times of theirs coming vehicles passage. Then, it calculates the waiting time and the remaining time if there is disturbance.

c) Utility based agent: It is used to generate high-quality behavior. In this agent group, there are many action sequences lead to desired goal. But some actions are better than others. Hear, if goal means success then utility means the degree of success.

- Regulator agent: Each vehicle has regulator agent that continuously receives the criteria values calculated by the agent's punctuality, regularity and correspondence. Then the regulator calculates the detection function to detect disturbance. Obviously, if there is a disturbance, it asks the associating criteria agents to compute theirs criteria values with remaining time. After receiving all criteria values, it optimizes the regulation action. This phase gives as output a sorted list of feasible regulation maneuvers. This optimization is based on the utility function to explain how successful it is? When there is a new fact, the human agent updates knowledge base of regulator agent by inserting new rules.

d) Learning agent: agents have to remember outcomes and generalize to future situations.

- Human agent: It percepts a sorted list of feasible regulation actions from regulator agent and it has to choose the suitable maneuver. Before that, it should respect the performance standard, because the regulator doesn't tell him about its success/failure. So, on each chosen solution, it should be responsible for improving its behavior with experience by updating a knowledge base of regulator. Consequently, when we have a new situation (not familiar incident, new traffic parameter...), it can suggest actions to come up with new and informative experiences.

2) Interaction Level: This part explains how agents interact with each other indirectly by acting on the environment or directly by communication. This interaction is ensured by protocols to govern the communication into the system. These protocols are used to define a sequence of messages between agents and to describe how agents should react when it receives messages. We can classify the regulation of public transport in interaction situation requiring certain coordination. This coordination is defined by a set of agent behaviors, objectives (finding the best regulation action) and skills (know-how of the regulator). In addition, it should take into account the resources available (roads, passengers, time ...) and the current traffic state.

The three main interaction components allow us to produce the typology of our interaction situations and characterize our system by these properties:

- Compatibility of goals: All agents have the same goal that finding the best regulation action for the public transport, 
- Competence of agents: All agents don't have sufficient skills. They should collaborate and coordinate to accomplish their main goal.

- Insufficient resources: the nature of the multimodal public transport domain (the multitude, uncertainty and the large amount of information) obliges agents to make shift with existing information to produce its best action.

The complex cooperation assumes that agents have to coordinate their actions in order to have the synergy of their skills. Our system presents the most complex of cooperative situations, because it adds to the allocation problems of coordination tasks the problems due to limited resources. Based on the classification of interaction situations presented by Jacques Ferber [14], the interaction of our agents is within the scope of the coordinated cooperation. We describe the interaction protocols of our system by using the following sequence diagram (see Fig. 5).

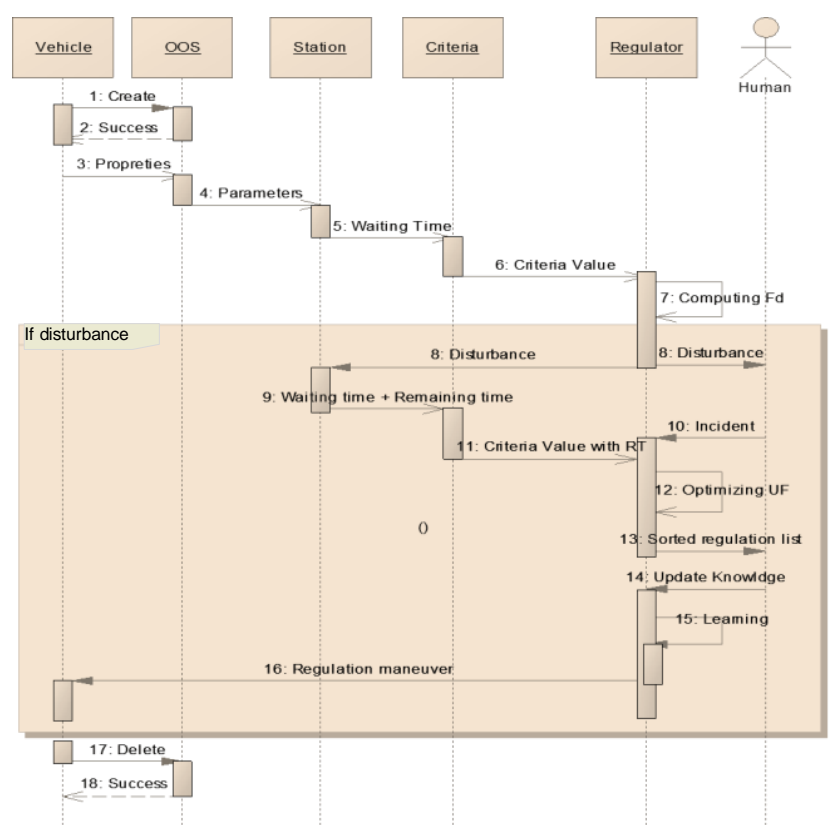

Fig. 5. Interaction protocols of RSSPT

\section{EXPERIMENTATION AND RESULT}

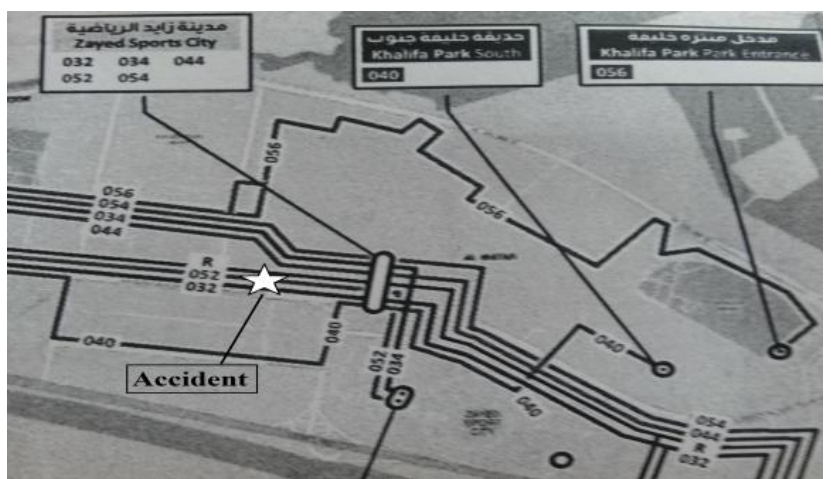

Fig. 6. Traffic Network zone of perturbation scenario.

We tested the regulation strategy of our system on the existing transportation system in Abu Dhabi. There is a list of regulation actions can be used to handle the public transport traffic. This list is classified into two groups: regulation action at terminus and regulation online [3], [7].
We consider scenario that shows perturbation detected after delay for lines 032 and 052 on Zayed Sports City station. This delay is due to an accident on the embassy area (see Fig. $6)$. The distributed weights are fixed on Table I.

TABLE I: BUSSES INFORMATION AT ZAYED SPORT CITY

\begin{tabular}{llllllll} 
Line & Freq. & $\begin{array}{l}\text { Theoretical time } \\
\text { at Station }\end{array}$ & $\begin{array}{l}\text { Detecting } \\
\text { perturb. time }\end{array}$ & $\Delta R t$ & $W r$ & $W p$ & $W c$ \\
\hline 032 & 20 & $05 \mathrm{~h}: 42 \mathrm{pm}$ & $05 \mathrm{~h}: 47 \mathrm{pm}$ & 22 & 0.4 & 0.4 & 0.2 \\
052 & 20 & $05 \mathrm{~h}: 38 \mathrm{pm}$ & $05 \mathrm{~h}: 43 \mathrm{pm}$ & 17 & 0.3 & 0.3 & 0.4 \\
\hline \hline
\end{tabular}

The two lines have at station four correspondences busses. All $f_{i}$ values are presented in Table II. We note that when busses have the same direction in the transfer station, the correspondence factor value is zero.

TABLE II: DISTRIBUTION OF FACTORS VALUES

\begin{tabular}{lllllll}
\hline \hline \multicolumn{7}{c}{ Factor Values $f_{\mathrm{i}}$ for lines } \\
\hline Line & 032 & 034 & 040 & 044 & 052 & 054 \\
032 & & 0.40 & 0.20 & 0.00 & 0.4 & 0.00 \\
052 & 0.25 & 0.00 & 0.20 & 0.30 & & 0.25 \\
\hline \hline
\end{tabular}

The system starts its optimization phase by the initial utility function value $U F_{\text {start }}$. The regulator of each bus wants to find the optimal regulation action with $U F_{\text {opt }}$ value. Note that the $U F_{\text {before }}$ value corresponds to theoretical value before perturbation.

TABLE III: DECISION AFTER OPLIMIZATION PHASE

\begin{tabular}{lll}
\hline \hline Line & U-turn at station & Save \\
\hline 032 & $06 \mathrm{~h}: 03 \mathrm{pm}$ & $6 \mathrm{mn}$ \\
052 & $06 \mathrm{~h}: 00 \mathrm{pm}$ & $11 \mathrm{mn}$ \\
\hline \hline
\end{tabular}

TABLE IV: RESULT VALUES FOR REGULATION PROCESS

\begin{tabular}{|c|c|c|c|c|c|c|c|c|c|c|}
\hline \multirow[b]{2}{*}{. } & \multicolumn{2}{|l|}{$V r$} & \multicolumn{2}{|l|}{$V p$} & \multicolumn{2}{|l|}{$V c$} & \multicolumn{4}{|c|}{ Utility Function } \\
\hline & 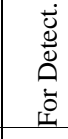 & $\begin{array}{l}\dot{a} \\
\text { ô } \\
\dot{0}\end{array}$ & 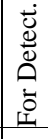 & 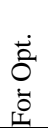 & 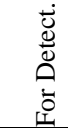 & $\begin{array}{l}\dot{\tilde{0}} \\
\dot{0} \\
\dot{0} \\
\dot{0}\end{array}$ & $L^{2}$ & 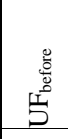 & $5^{\frac{5}{\frac{5}{5}}}$ & $S^{\stackrel{\overrightarrow{0}}{0}}$ \\
\hline 32 & 25 & 39 & 5 & 24 & 13 & 23 & 4.61 & 2.61 & 17.77 & 14.21 \\
\hline 52 & 25 & 31 & 5 & 11 & 16.3 & 22.5 & 8.03 & 2.52 & 17.13 & 12.23 \\
\hline
\end{tabular}

Following optimization phase, the system recommends that the better action regulation is a U-turn of the opposite direction bus for the two disturbed busses (see Table III and IV).

These results show that the utility function is a good way for optimization. Also, the optimization function used by our MAS strategy improves the traffic of public transport. As we can see, that our system detect and regulate simultaneously several perturbations. Also it optimizes the utility function on many criteria. In addition it can ameliorate its knowledge base by updating its list of regulation actions.

\section{CONCLUSION AND PERSPECTIVES}

We have proposed a Multi-agent strategy for Regulation Support System of Public Transport (RSSPT). Unlike other works, this strategy shows that our system does lead to ensure the two phases of regulation such as detection of perturbation and regulation process. As well as, our model takes into account three criteria: punctuality, regularity and 
correspondence.

The objective of this research is to improve the passenger quality service of public transport. In our approach, regulation of public transport is distributed and obtained thanks to communication and collaboration between heterogeneous agents. Firstly, we demonstrated that existing approaches of support system regulation have several limits. Secondly, we presented the regulation process for making decision. Thirdly, we explained all mathematical formulas used in our optimization problem. Fourthly, we presented how we used multi-agents approach for modeling and we described the agent technology in the three levels: organization, intra-agent and interaction. Finally, we presented simulation and result of perturbation scenario on a real traffic network.

On a perspective, we propose framework that merges common design characteristics of criteria agents. That is allows us to operate the system not only with punctuality, regularity and correspondence agent, but with criteria that can be adjusted following to the company's needs like pollution, economy, etc. We aim to tackle the issue of our RSSPT design at this level. The deeper validation is in progress by using a multi-agents platform JADE.

\section{REFERENCES}

[1] B. Karim, T. Bonte, M. Sevaux, and C. Tahon, "SART: un système d'aide à la décision pour la régulation d'un réseau de transport bimodal," LAMIH/SP, UMR-CNRS 8530, 2005.

[2] T. Cazenave1, F. Balbo1, and S. Pinson, "Monte-carlo bus regulation," presented at 12th International IEEE Conference on Intelligent Transportation Systems, October 2009.

[3] B. Chen and H. H. Cheng, "A review of the applications of agent technology in traffic and transportation systems," IEEE Tansactions on Intelligent Transportation Systems, vol. 11, no. 2, June 2010.

[4] N. Bhouri, F. Balbo, and S. Pinson, "An agent-based computational approach for urban traffic," Springer-Verlag, 2012.

[5] H. Sofiane and B. Neïla, "Régulation du trafic urbain avec une priorité aux transports en commun a l'aide d'un système multi-agents," presented at $8 \mathrm{e}$ Conférence Internationale de MOdélisation et SIMulation, 2010.

[6] B. Neila and P. Lotito, "An intermodal traffic control strategy for private vehicule and public transport," presented at 10th Euro Working Group on Transportation Poznan Poland, 2005.

[7] Z. Salah, "SARR: Système d'Aide à la Régulation et la Reconfiguration des Réseaux de Transport Multimodal," 2007.

[8] K. Sofiene, "Commande et optimisation pour la régulation du traficurbain multimodale sur de grands réseaux urbains, " 2010.

[9] F. Balbo and S. Pinson, "Dynamicmodeling of a disturbance in a multiagent system for traffic regulation," Decis. Support Syst., vol. 41, no. 1, pp. 131-146, 2010.

[10] Laichour, "Modélisation Multi-agent et aide à la décision: application à la régulation des correspondances dans les réseaux de transport urbain," Universite de Lille 2002.
[11] N. Morri, S. El Hadouej, and L. B. Said, "Multi-agent optimization model for multi-criteria regulation of multi-modal public transport," NNGT Int. J. on Artificial Intelligence, vol. 3, June 2015.

[12] F. Balbo and S. Pinson, "Using intelligent agents for transportation regulation support system design," Transp. Res. Part C Emerg. Technol., vol. 18, no. 1, pp. 140-156, 2010.

[13] A. L. Bazzan, "Opportunities for multi-agent systems and multiagent reinforcement learning in traffic control," Auton. Agents Multi Agent Syst., vol. 18, no. 3, pp. 342-375, 2009.

[14] J. Ferber, "Les Systèmes Multi gaents, vers une intelligence collective," Inter Edition, 1995.

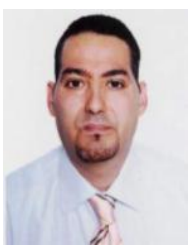

Nabil Morri was born 1974 in Gafsa, Tunisia. He received his master degree in computer science from ENSI of Mannouba University in Tunisia and he has an aggregation for teaching from Ministry of High Education and Technology of Tunisia since 2001. He obtained a certificate of pedagogic qualification from Cegep Levi-Lozon of Canada in 1998.

Currently he is a senior lecturer in computer science at Emirates College of Technology. His research area is in artificial intelligence and multi-agent system. He is registered in $\mathrm{PhD}$.

$\mathrm{He}$ is also a member in INSTICC (Institute for System and Technologies of Information, Control and Communication) and SOIE research laboratory. He published papers in indexed international conferences, journals and workshop. He serves as a chair of some sessions in international conference.

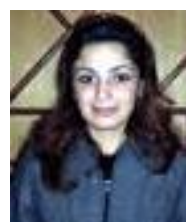

Sameh Hadouaj was born on 1974 in Tunis, Tunisia. She is an assistant professor in computer science at Higher College of Technology in Abu Dhabi. She received her master degree in artificial intelligence and master degree and $\mathrm{PhD}$ degree in computer science from Paris 6 University.

Since 2005, she is a member of SOIE Research Laboratory of Tunis University. Her researches lie in the general field of artificial intelligence with special focus on multi agent social simulations and complex systems. She has supervised many master and PHD students. The subject studies turn around robotic simulation, e-government, pedestrian simulation, road traffic regulation and pastoral dynamic.

She published over 20 papers in indexed international conferences, journals and books. She also serves as a program committee member for various international conferences and workshops.

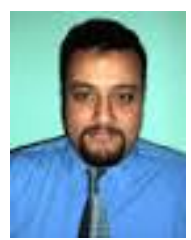

Lamjed Ben Said was born on 1975 in Tunis, Tunisia. He has received the B.S. degree in computer science applied to management from the High Institute of Management of Tunis, University of Tunis, Tunisia, in 1998. He has obtained the M.S. degree and Ph.D. degree in computer science from the University of Paris VI, France, in 1999 and 2003 respectively.

He was a research fellow for three years at France Telecom (R\&D). Currently he is a director of the High Institute of Management of Tunis (Tunisia). He is now also the scientific coordinator of Optimization Strategies and Intelligent Computing laboratory (SOIE) (Tunisia) and the general secretary of the Tunisian Association of Artificial Intelligence. His research interests cover multi-agent systems, multi-criteria decision making, evolutionary computation, supply chain management and behavioral economics. 